P284 A QUESTIONNAIRE STUDY OF ELECTRONIC CIGARETTE USAGE IN PATIENTS ATTENDING RESPIRATORY CLINICS IN A DISTRICT GENERAL HOSPITAL

AD Macfoy, E Crawford, K Srinivasan, H Moudgil. University of Keele Medical School, Staffordshire and Shrewbury and Telford Hospitals NHS Trust, Shropshire

\subsection{6/thoraxjnl-2014-206260.402}

Background/objectives In the UK there are now more than two million users and more than 400 variations on electronic cigarettes (e-cigarettes) based on nicotine strength, flavours, devices etc. Despite the exponential rise in the use of e-cigarettes primarily as an adjunct to quit smoking strategies, the drive has predominantly been patient led and industry marketed with medical profession reluctant to engage, citing potential toxic effects as yet uncertain. Reporting from semi-rural community and focusing on respiratory patients attending respiratory clinics, objectives were to (1) document the current smoking pattern of our patients, (2) investigate their prior health seeking behaviour with respect to quit smoking, and (3) more specifically with respect to e-cigarettes address some of the questions raised with respect to where the medical profession may still have a role.

Methods Prospective, self-completed, questionnaire based survey of patients $(>75 \%)$ attending respiratory clinic first three weeks July 2014,

Results Of 78 patients, mean ((range) age was 63 (17-91) years with $49 \%$ male. Of these, 17 were smokers, 32 ex-smokers, and 29 never smokers. $42 / 49$ (86\%) had previously attempted to quit smoking; 26/42 had used no outside support, two had used nicotine gum or patches, three used drug therapies including Zyban or Champix, seven had used a combination, and four had used other unspecified techniques. 11/49 (22.4\%) of those who had ever smoked had tried e-cigarettes: average set up was $£ 23.33$ with purchase on-line for three, specialist shops for four, market stalls for two, supermarket for one, and for one patient it was a gift. Only one patient had prior concerns about harmful effects, with two others asking and two others specifically being told by their retailer. Similarly, only two were given advice about suitable dosing based on baseline nicotine use, and two others about how to plan use and weaning.

Conclusions Although based on a small number of patients, the high use of e-cigarettes is recognised as is the intention to quit smoking. Importantly, the survey identifies a need for patient education about use and potential for harm and it is important that we now actively engage.

\section{P285 ASSESSING THE IMPACT OF VARENICLINE INITIATION DURING ACUTE HOSPITAL ADMISSION FOR CURRENT SMOKERS WITH RESPIRATORY DISEASES: 18-MONTH EXPERIENCE FROM AN INNER CITY DISTRICT TEACHING HOSPITAL}

A Ainley, E Pang, B Coleman, M Stern, LJ Restrick. Department of Respiratory Medicine, Whittington Health NHS Trust, London, UK

\subsection{6/thoraxjnl-2014-206260.403}

Introduction Smoking is a significant cause of respiratory disease and risk factor for chronic obstructive pulmonary disease (COPD) and asthma admissions. 70\% of smokers admitted to hospital want to quit and quit smoking interventions during acute admission are NICE recommended. ${ }^{1}$ Many patients with respiratory disease are highly nicotine-dependent and varenicline is an effective treatment ${ }^{1,2}$ but is not routinely initiated during admission.
Method We retrospectively reviewed the notes of all patients prescribed varenicline during in-patient stay on the respiratory ward over 18 months (August 2012-January 2014). Baseline data included demographics, disease details (diagnosis, spirometry) and smoking history (tobacco/cannabis use, pack/joint-years). The primary outcomes were carbon monoxide (CO) validated quit rates at 4-weeks and self-reported quit rates at 6-months and 1-year.

All patients were seen on the ward by a smoking cessation advisor and after discharge as per NICE guidance. ${ }^{1}$ Nicotine withdrawal during varenicline initiation was treated with standard combination nicotine replacement therapy. ${ }^{1}$

Results 44 patients (17M:27F) were prescribed varenicline during admission. Mean (range) age was 61 (23-81) years with median (range) $50(8-180)$ pack-years. 8/44 (18\%) also smoked cannabis. 29 (66\%) had COPD, 7 (16\%) asthma, and 8 (18\%) had both. Mean (SD) $\mathrm{FEV}_{1}$ was $1.18(0.52) \mathrm{L}(\mathrm{n}=40)$ with $\mathrm{FEV}_{1} \%$ predicted $47(21) \%(\mathrm{n}=26) .7$ patients $(16 \%)$ died; all from smoking-related diseases, within 18 months of admission with mean (range) age at death $71(61-78)$ years. 2 were lost to follow-up. CO-validated 4-week quit rate was 48\% (21/44). Selfreported 6-month and 1-year quit rates were 41\% (18/44) and $20 \%$ (9/44) respectively. Only 4/44 (9\%) stopped varenicline early due to side-effects (nausea/headache).

Conclusion Varenicline was safe and well-tolerated when initiated in hospital. The 4-week 48\% quit rate for these 'sick' smokers was almost as high as the $52 \%$ national target for 'well' smokers. Self-reported 6-month quit rates were almost as good as the best published rates with intensive support in COPD $(41 \%$ cf $49 \%) .^{2}$ Varenicline should be used as a treatment for smokers admitted with respiratory disease. ${ }^{1}$

\section{REFERENCES}

1 NICE PH guidance 48. Smoking cessation in secondary care. 2013

2 Jiménez Ruiz et al. Nicotine and Tobacco Research. 2012;14(9):1035-1039

P286 RECOMMENDATIONS FOR SMOKING CESSATION
SERVICE PROVISION FOR SMOKERS WITH COPD WITH
MULTIPLE COMPLEX NEEDS: FINDINGS FROM A PILOT
STUDY

SY Yap, E Pang, S Lunn, C Croft, M Stern. Whittington Health, London, UK

10.1136/thoraxjnl-2014-206260.404

Introduction Smokers with COPD are highly nicotine addicted and often have additional complex needs. Quit rates are poor and there is little evidence-based guidance on specific cessation interventions for these patients. This pilot study aimed to identify barriers to smoking cessation for this patient group.

Method Smokers with COPD were offered up to 12 individual sessions with a clinical psychologist in addition to standard smoking cessation counselling and pharmacotherapy. The psychological intervention included an initial assessment and formulation on factors maintaining smoking which informed an individualised psychology intervention targeting barriers to smoking cessation.

Results 57 patients (moderate COPD, high prevalence of complex physical andpsychological comorbidities) were included in the study (Table 1). 20/57 (35\%) patients attended $>2$ sessions (mean $=5$, range $2-12$ ). $7 / 20$ had already quit (relapse prevention referrals), 13 were smokers. 22/57 (39\%) patients never engaged, $15 / 57$ (26\%) were lost to follow-up. 6/7 (86\%) of the relapse prevention group maintained their quit. 2/13 (15\%) of 